\title{
Meyen, Michael: Das Erbe sind wir. Warum die DDR-Journalistik zu früh beerdigt wurde. Meine Geschichte.
}

\author{
Köln: Herbert von Halem 2020. 372 Seiten. Preis: $€ 28,00$
}

\section{Martin Löffelholz}

Angenommen: 3. Juni 2021 / Online publiziert: 24. Juni 2021

(C) Der/die Autor(en) 2021

Als Maria Löblich fragte, ob ich Michael Meyens neues Buch rezensieren möchte, war ich zunächst skeptisch: Arbeitszeit verplempern mit der Lektüre autobiographischer Notizen eines Kollegen, der Rechtsideologen wie Ken Jebsen Interviews gibt oder die bundesdeutsche Pandemie-Öffentlichkeit in „Rubikon“ mit der Kommunikation zwischen „Regierenden und Regierten“ in der DDR vergleicht? Zugesagt habe ich dann doch, denn nach Meyens früheren Arbeiten zum Journalismus in der DDR reizte es mich herauszufinden, wie er seine titelgebende These, dass die „DDR-Journalistik zu früh beerdigt wurde“, begründen würde.

Einfach macht es Meyen seinen Leser*innen allerdings nicht. Weder folgt er dem traditionellen Aufbau wissenschaftlicher Werke, noch stringent seiner Ausgangsthese, dass das mit der Leipziger Sektion Journalistik ,entsorgte“ Paradigma, ,heute helfen könnte, die Redaktionen aus der Umklammerung der Politik zu befreien oder von den Zwängen einer kommerziellen Medienlogik“ (S. 8). Wer die narratologische Melange primärer und sekundärer, diegetischer und nichtdiegetischer Schilderungen entwirrt, findet in den zehn Kapiteln drei wesentliche Erzählstränge: Einblicke in die Entwicklung der Sektion Journalistik, eine eher provokante als anspruchsvolle Kritik am „hegemonialen DDR-Diskurs“ (S. 23, 28, 34, 60) sowie „eine persönliche Geschichte [...], die so unerhört ist und so spannend, dass sie ein ganzes Buch trägt“" (S. 16).

Diese Selbsteinschätzung mag anmaßend erscheinen. Wer von der eigenen Geschichte jedoch dermaßen überzeugt ist, sollte sie aufschreiben, finde ich. Anders sah das Meyens früherer Kollege Heinz Pürer, der ihm von dem Buch abgeraten habe, da er ,viel zu nah dran“ (S. 27) sei. Man werde ihm vorwerfen, sich selbst

Prof. Dr. Martin Löffelholz ( $₫)$

Institut für Medien und Kommunikationswissenschaft, Technische Universität Ilmenau,

Ehrenbergstraße 29, 98693 Ilmenau, Deutschland

E-Mail: Martin.Loeffelholz@tu-ilmenau.de 
aufwerten zu wollen. „Ja, lieber Heinz: Das will ich.“ (S. 27) - und so finden sich in dem Buch diverse autobiographische Fragmente, die uns Meyens Kindheit auf Rügen, sein Praktikum bei der Ostsee-Zeitung oder seine 34 Monate beim Wachregiment des Ministeriums für Staatssicherheit näher bringen. Dass der junge Meyen „Parteijournalist werden wollte“ (S. 95) und in den Umbruchjahren 1989/90 eher indifferent agierte, erscheint vor diesem Hintergrund folgerichtig, jedoch - folgt man Steffen Maus (2019) lesenswerter Analyse über das „Leben in der ostdeutschen Transformationsgesellschaft“ - keineswegs so ,unerhört“, wie Meyen autoexplikativ annimmt.

Weniger transparent beschreibt Meyen hingegen das, was Mau „habituelle und kulturelle Akkommodation“" nennt. Nach Diplom, Promotion und Habilitation am neuen Leipziger Institut für Kommunikations- und Medienwissenschaft erhält Meyen 2001, gefördert von Brosius und Donsbach, einen Ruf nach München. Dort macht er die DDR-Journalistik zu seinem Thema - und wandelt sich zu einem Kritiker des „,hegemoniale[n] Diskurs[es], der aus der DDR partout eine ,Diktatur“ machen will“ (S. 116). Gestützt durch „Leitmedien, die aus der Universität Leipzig eine Kaderschmiede der SED machen und dabei auch auf die Sektion Journalistik zielen“" (S. 279), habe der hegemoniale Diskurs einen Vertrauensverlust der Medien befördert, weil er „ostdeutsche Journalisten in aller Regel in Nischen verbannt und sich so lange Zeit selbst verstärkt hat" (S. 34). Abgesehen von Randnotizen, etwa zu Gramscis Hegemonie- oder Mouffes Diskursbegriff, werden die unterstellten Wirkungszusammenhänge jedoch weder theoretisch noch empirisch untermauert.

Hätte Meyen die Diskurstheorie von Laclau und Mouffe auf seine (auto-)biographischen Erzählungen bezogen, wäre aus Einzelerinnerungen vielleicht eine elaborierte Analyse zur diskursiven Konstituierung individueller Identitäten in der Sektion Journalistik entstanden. Stattdessen bleibt Meyen nah an den erinnerten Biografien von Lehrenden und Studierenden, ohne jedoch seine Methodik genauer offenzulegen. Aus der Deutung biographischer Sinnkonstruktionen, ergänzt durch Archivarbeit, konstruiert er ein mosaikartiges Bild einer Einrichtung, in der etwas ,trotz Parteiherrschaft gewachsen war: Geschichte des Journalismus [...], ,Ansätze zu einer Theorie der sozialen Kommunikation", die Inhaltsanalyse, die [...] so nah dran war an der Medienrealität, dass die Partei die Befunde im Panzerschrank verschloss, die Arbeiten zur Stilistik, die Horst Pöttker später in Dortmund vor dem Vergessen rettete, und der ,journalistische Schaffensprozess““( (S. 90).

Dass in der Sektion Journalistik substantielle Beiträge zu einer wissenschaftlich begründeten Ausbildung von (Partei-)Journalisten entstanden sind, steht für mich seit meiner Leipziger Zeit (1994-1998) außer Frage. Insofern ist es schade, dass Meyen weder diese Leistungen genauer darstellt, noch die Historie der Sektion Journalistik systematisch nachzeichnet. Manche Passagen, etwa zur Rolle von Hermann Budzislawski, der 1948 aus den USA in die ein Jahr später gegründete DDR wechselt, zur IAMCR-Konferenz 1974 in Leipzig oder zu den , Überlegungen der Alternativgruppe zu einer Sektionsreform“ vom 25. Januar 1990, die in der Forderung nach einer „umfassenden demokratischen Öffentlichkeit“ (S. 271) kulminieren, sind erhellend, driften jedoch oft ins Anekdotische.

Zusammengenommen ist mir allerdings nicht klar geworden, warum Meyen ,das ,rote Kloster ' in Leipzig, der Prototyp einer Schule für Parteijournalisten, als Lö- 
sung für die Medienkrise der Gegenwart“ (S. 13) betrachtet. Nachvollziehbar finde ich zwar das Argument, gerade die Gängelung durch die SED habe „ein Journalismusideal gefüttert, das ,Öffentlichkeit als gesellschaftlichen Auftrag 'sieht.“ (S. 8) Dieses Ideal, entwickelt u. a. von meiner geschätzten früheren Kollegin Sigrid Hoyer in den Überlegungen zu einer Sektionsreform, ist aber erst ,,auf den Ruinen der DDR [...] gewachsen“ (S. 324), wie Meyen einräumt.

So hinterlässt der Band einen ambivalenten Eindruck - zum einen theoretisch kaum elaboriert, methodisch nicht immer transparent, stilistisch eher erzählerisch, missionarisch im Duktus. Auf der anderen Seite sind es gerade die erzählerischen Elemente, die jenen, für die die DDR eine blackbox war (und ist), zumindest ansatzweise zeigen, dass in der Leipziger Sektion Journalistik auch Menschen tätig waren, ,die ihre Arbeit so gut wie möglich machen wollen. Menschen, die sich streiten, die erzählen, was sie in den Seminaren erlebt haben, die das einbringen, was sie aus den Redaktionen hören. Menschen, die dabei den Sozialismus und das Leben im Land besser machen wollen“" (S. 211).

Funding Open Access funding enabled and organized by Projekt DEAL.

Open Access Dieser Artikel wird unter der Creative Commons Namensnennung 4.0 International Lizenz veröffentlicht, welche die Nutzung, Vervielfältigung, Bearbeitung, Verbreitung und Wiedergabe in jeglichem Medium und Format erlaubt, sofern Sie den/die ursprünglichen Autor(en) und die Quelle ordnungsgemäß nennen, einen Link zur Creative Commons Lizenz beifügen und angeben, ob Änderungen vorgenommen wurden.

Die in diesem Artikel enthaltenen Bilder und sonstiges Drittmaterial unterliegen ebenfalls der genannten Creative Commons Lizenz, sofern sich aus der Abbildungslegende nichts anderes ergibt. Sofern das betreffende Material nicht unter der genannten Creative Commons Lizenz steht und die betreffende Handlung nicht nach gesetzlichen Vorschriften erlaubt ist, ist für die oben aufgeführten Weiterverwendungen des Materials die Einwilligung des jeweiligen Rechteinhabers einzuholen.

Weitere Details zur Lizenz entnehmen Sie bitte der Lizenzinformation auf http://creativecommons.org/ licenses/by/4.0/deed.de.

Prof. Dr. Martin Löffelholz ist Leiter des Fachgebiets Medienwissenschaft am Institut für Medien- und Kommunikationswissenschaft der Technischen Universität Ilmenau. 